\title{
Serial Recall among Adolescents in Abakaliki: Examining the Roles of Reproductive Maturity and Age
}

\author{
Chiedozie Okechukwu Okafor ${ }^{1, *}$, Emmanuella U. Anozie ${ }^{1}$, Sorochi Berny Anyanwu ${ }^{1}$ \\ ${ }^{1}$ Department of Psychology, Alex Ekwueme Federal University \\ Ndufu-Alike, Ebonyi State, Nigeria \\ *Corresponding author's email: chiedozie.okafor [AT] funai.edu.ng
}

\begin{abstract}
The study investigated the roles of reproductive maturity and age on serial recall. Eighty (80) senior secondary school students from a public community secondary school in Abakaliki participated in the study. Participants comprised younger and older adolescents within the ages of $11-13$ years with a mean of 12.25 years, and $14-17$ years with a mean age of 15.73 years, respectively. The design of the study is quasi-experimental; the participants responded under 4 independent conditions: younger participants with reproductive maturity and younger participants without reproductive maturity; older participants with reproductive maturity and older participants without reproductive maturity. Data analysis using $2 \times 2$ ANOVA (F-test) revealed significant influence of reproductive maturity; nonsignificant influence of age, and significant interaction of reproductive maturity and age on serial recall. The results were discussed in terms of their relevance in education and eye-witness testimony.
\end{abstract}

Keywords--- Reproductive maturity; Age; Serial recall; Female; Adolescence

\section{INTRODUCTION}

Recall of events in order in which they are presented has been a problem in forensic science especially crime scene recall, eyewitness testimony and criminal identification. Despite research results favouring women face recognition and recall of events [1], adolescent girls who experience rape have had problems representing the situation or even identifying their assailants [2]. Rhodes, Greene and Naveh-Benjamin [3] presented a meta-analysis of 36 articles reporting 89 direct comparisons of free recall and recognition performance in younger and older groups of participants and found significant age differences; indicating the younger people recalled better than older people irrespective of the age gap. However, Brinley analyses of the data suggest that distinct functions are needed to relate younger and older performance for recall, hence the present study.

Previous research confirmed that women are superior in face recognition ([1]. Females also outperformed males in recalling everyday tasks [2], stories [4], names [5], and episodic memories [6, 7]. Apart from the limitations placed on memory by heightened emotion [2], this study contends that reproductive maturation could be a factor in recall especially serial recall. Hence, such hormones as estrogen may have significant influence on female intelligence. To the best of our knowledge, no such study has ever been conducted. Therefore, this study was set to investigate the following research questions: Will reproduction maturity i.e. menstrual status, significantly influence serial recall? Will Age significantly influence serial recall? Will there be any significant interaction between Age and Reproductive maturity on Serial Recall?

The purpose of the study is to ascertain the role of the Reproductive maturity and Age on serial recall. The result of this research will hopefully throwing more light on the influence of age and reproductive maturation on intellectual ability. Initially, people may believe that once you are above a particular age, you are expected to think hypothetically and your intellectual ability must have developed and yes, true but it could also be that reproductive maturation, associated with the onset of the production of hormones such as estrogen, can be a sort of moderating factor, in the sense that it could either be in favor or not of our special intellectual abilities.

In this study, reproductive maturity refers to the Menstrual Status of an adolescent female. Those with reproductive maturity are refers to here as those who have started seeing their menstrual cycle while those who are not mature are those who have not started seeing their cycle. Age refers to the period of time someone has been alive or something has existed. Serial Recall is the ability to recall items or events in the order in which they occurred. A serial recall task requires participants to recall a list of items in a specific order, usually the order in which they were presented. The ability of humans to store items in memory and recall them is important to the use of language. Imagine recalling the different parts of a sentence, but in the wrong order. The ability to recall in serial order has been found not only in humans, but in a number of nonhuman primate species and some non-primates. 
The following hypotheses were tested:

Hypotheses 1- reproductive maturity will not significantly influence serial recall among adolescents

Hypotheses 2- age will not significantly influence serial recall among adolescents

Hypotheses 3- there would be no significant interaction of age and reproductive maturity on serial recall among adolescents.

\section{PARTICIPANTS}

Eighty (80) female adolescents participated in the study. They comprised secondary school students from a public community secondary school in Abakaliki, Ebonyi State, Nigeria. Forty (40) of the participants were between the ages of 11 and 13years (younger age) with a mean age of 12.25 years, and the other 40 were between the ages of 14 and 17 years (older age) with a mean age of 15.73 years. Using Simple Random Sampling Method, reproductively "ripe" and "unripe" participants in each of the two age groups were drawn from a pool of 200 females in senior classes of the school, thus making a 4-independent group thus: Younger participants with reproductive maturity and younger participants without reproductive maturity; older participants with reproductive maturity and older participants without reproductive maturity.

\section{STIMULUS MATERIALS}

Stopwatch timer

80 pieces of blue pen (Bic)

80 pieces of plain sheets of white paper (A4)

A large classroom

List of 20 words containing names of persons, objects, events and time (80 copies) (see Appendix).

\section{PROCEDURE}

The participants were approached on a school day in their various class rooms with the assistance of the Dean of studies. They were told of the rationale behind the study as purely academic and was assured of the confidentiality of their responses. The main purpose of the study was hidden form them before the experiment as a measure of control. Rather they were told that the study was on global health challenge among the female adolescents. We identified the participants using a demographic questionnaire instrument in which they were asked to give some information about their current status such as age, academic level (class), year of first menstrual cycle (if any), after volunteering to participate. Their informed consent was obtained after creating rapport.

Two hundred (200) copies of the demographic questionnaire were distributed to all the female students in senior secondary classes after given them serial number tags. They were given 10 minutes to return the filled copies of the questionnaire which they all did. Out of the 200 copies distributed, 96 were between the ages of 11 and 13 years (younger age) while 104 were between the ages of 14 and 17 years (older age). Out of the 96 (younger age), 29 had started experiencing menstrual period while 67 had not; while out of 104 (older age), 71 had started experiencing menstrual period while 33 had not.

Using a simple random sampling, 20 participants were drawn from each of the 4 groups (Younger participants with reproductive maturity and younger participants without reproductive maturity; older participants with reproductive maturity and older participants without reproductive maturity); producing a sample of 80 experimental participants. They were identified using the serial number tags given them before the distribution of the demographic questionnaire.

In drawing the sample, we made us of the lucky-dip technique. Thus, in drawing younger participants with reproductive maturity, they were presented with 29 pieces of wrapped comprising 20 YES and 9 NO; those who picked YES were included group A. For younger participants without reproductive maturity, 67 pieces of wrapped paper with 20 YES and 47 NO were presented. Those who picked YES were included in group B. For older participants with reproductive maturity, 71 pieces of wrapped paper comprising 20 YES and 51 NO were presented. Those who picked YES were included in group C. And, for older participants without reproductive maturity, 33 pieces of wrapped paper comprising 20 YES and 13 NO were presented. Those who picked YES were included in group D. The sample was taken to a large class room where they were kept accordingly in 4 independent groups: group A, group B, group C \& group D respectively.

The participants were then presented with the list of 20 words containing names of persons, objects, events and time, and were given 5 minutes to read and memorize the words. At the end of the 5 minutes, the list of words were withdrawn and each participant was given a pen and a plain sheet of white paper to recall, serially, the words she had memorized from the list. At the end of the study, the participants were called together and debriefed. Each participant was given a blue pen and a HB pencil as incentive for participation. 
The scoring was based on the number of words recalled in the order they were presented. All words written disorderly, incorrectly or not on the list were discarded. All words written orderly (even when some were omitted) were scored. Each word attracted 1 mark. The minimum score is 0 while the maximum score is 30 .

\section{DESIGN AND STATISTICS}

The design of this study is quasi-experimental. This is because the independent variables (reproductive maturity and age) were not manipulated in the study. Thus, we tested the dependent variable (serial recall) based on independent variables that had been manipulated by nature. The statistics for data analysis is Two-Way analysis analysis of variance (ANOVA). This is because the study is set to examine the interaction to the two independent variables with 2 factors each (Reproductive maturity: menstrual cycle and no menstrual cycle; Age: older and younger) on one dependent variable (serial recall) using the quasi-experimental method.

\section{RESULTS}

Table 1 : Mean and Standard deviation of reproductive maturity and age on serial recall

\begin{tabular}{lllll}
\hline Reproductive Maturity & Age & Mean & Std. Deviation & N \\
\hline \multirow{2}{*}{ Maturity } & & & & 20 \\
& Older adolescents & 3.5500 & 2.37254 & 20 \\
& Younger adolescents & 5.9500 & 2.85574 & 40 \\
No Maturity & Total & 4.7500 & 2.86222 & 20 \\
& Older adolescents & 7.2500 & 2.26820 & 20 \\
\multirow{2}{*}{ Total } & Younger adolescents & 5.7500 & 2.73140 & 40 \\
& Total & 6.5000 & 2.59190 & 40 \\
\hline Total & Older adolescents & 5.4000 & 2.95956 & 40 \\
\hline
\end{tabular}

The results of means and standard deviations in Table 1 show that participants who had reached reproductive maturity obtained at total mean of 4.75 on serial recall while participants who had not reached reproductive maturity obtained a total higher mean of 6.50 on serial recall. Older participants obtained a total mean of 5.40 on serial recall while younger participants obtained a relatively equal total mean of 5.85 on serial recall. However, a 2 X 2 ANOVA F-test was used to determine the significance of the observed mean differences.

Table 2: ANOVA summary of reproductive maturity and age on serial recall

\begin{tabular}{|c|c|c|c|c|c|c|c|c|}
\hline Source & $\begin{array}{l}\text { Sum of } \\
\text { Squares }\end{array}$ & df & $\begin{array}{l}\text { Mean } \\
\text { Square }\end{array}$ & $\mathbf{F}$ & Sig. & $\begin{array}{l}\text { Partial Eta } \\
\text { Squared }\end{array}$ & $\begin{array}{l}\text { Noncent. } \\
\text { Parameter }\end{array}$ & $\begin{array}{l}\text { Observed } \\
\text { Power }^{\text {b }}\end{array}$ \\
\hline Rep. Maturity & 61.250 & 1 & 61.25 & 9.28 & $0.003^{*}$ & 0.109 & 9.284 & 0.853 \\
\hline Age & 4.050 & 1 & 4.050 & 0.61 & 0.436 & 0.008 & 0.614 & 0.121 \\
\hline Rep. Maturity * Age & 76.05 & 1 & 76.05 & 11.52 & $0.001 * *$ & 0.132 & 11.527 & 0.918 \\
\hline Error & 501.40 & 76 & 6.597 & & & & & \\
\hline Total & 3174.00 & 80 & & & & & & \\
\hline Corrected Total & 75 & 79 & & & & & & \\
\hline
\end{tabular}

$* \mathrm{p}<0.05 ; * * \mathrm{p}<0.01$

Result of ANOVA F-test in Table 2 indicates significant influence of reproductive maturity on serial recall, $\mathrm{F}(1,76)=$ $9.28, \mathrm{p}<.05$. This means that there is a statistically significant difference between the reproductively mature participants and the none-mature participants on serial recall. Thus, hypothesis 1 was rejected. The result indicates non-significant influence of age on serial recall, $\mathrm{p}>0.05$. This means that there is no statistically significant difference between older and younger participants on serial recall. Thus hypothesis 2 was accepted. The result also indicates significant interaction of reproductive maturity and age on serial recall, $\mathrm{F}(1,76)=11.52, \mathrm{p}<.01$. This means that reproductive maturity and age jointly influence serial recall. 
Table 3: Mean and standard error of interaction of reproductive maturity and age on serial recall

\begin{tabular}{llcccc}
\hline \multicolumn{1}{c}{ Age } & Reproductive Maturity & Mean & $\begin{array}{c}\text { Std. } \\
\text { Error }\end{array}$ & \multicolumn{2}{c}{ 95\% Confidence Interval } \\
\hline & & & \multicolumn{2}{c}{$\begin{array}{l}\text { Lower } \\
\text { Bound }\end{array}$} & Upper Bound \\
& & & & 2.40 & 4.69 \\
Older & Maturity & 3.55 & .57 & 6.10 & 8.39 \\
adolescents & No maturity & 7.25 & .57 & 4.80 & 7.09 \\
Younger & Maturity & 5.95 & .57 & 4.60 & 6.89 \\
\hline adolescents & No maturity & 5.75 & .57 & & \\
\hline
\end{tabular}

Observation of result in Table 3 shows that all the 4 categories of participants had same standard error of .57 but the highest point for serial recall was obtained by older adolescents who were yet to attain reproductive maturity $(M=7.25)$ (see figure 1 for graph representation).

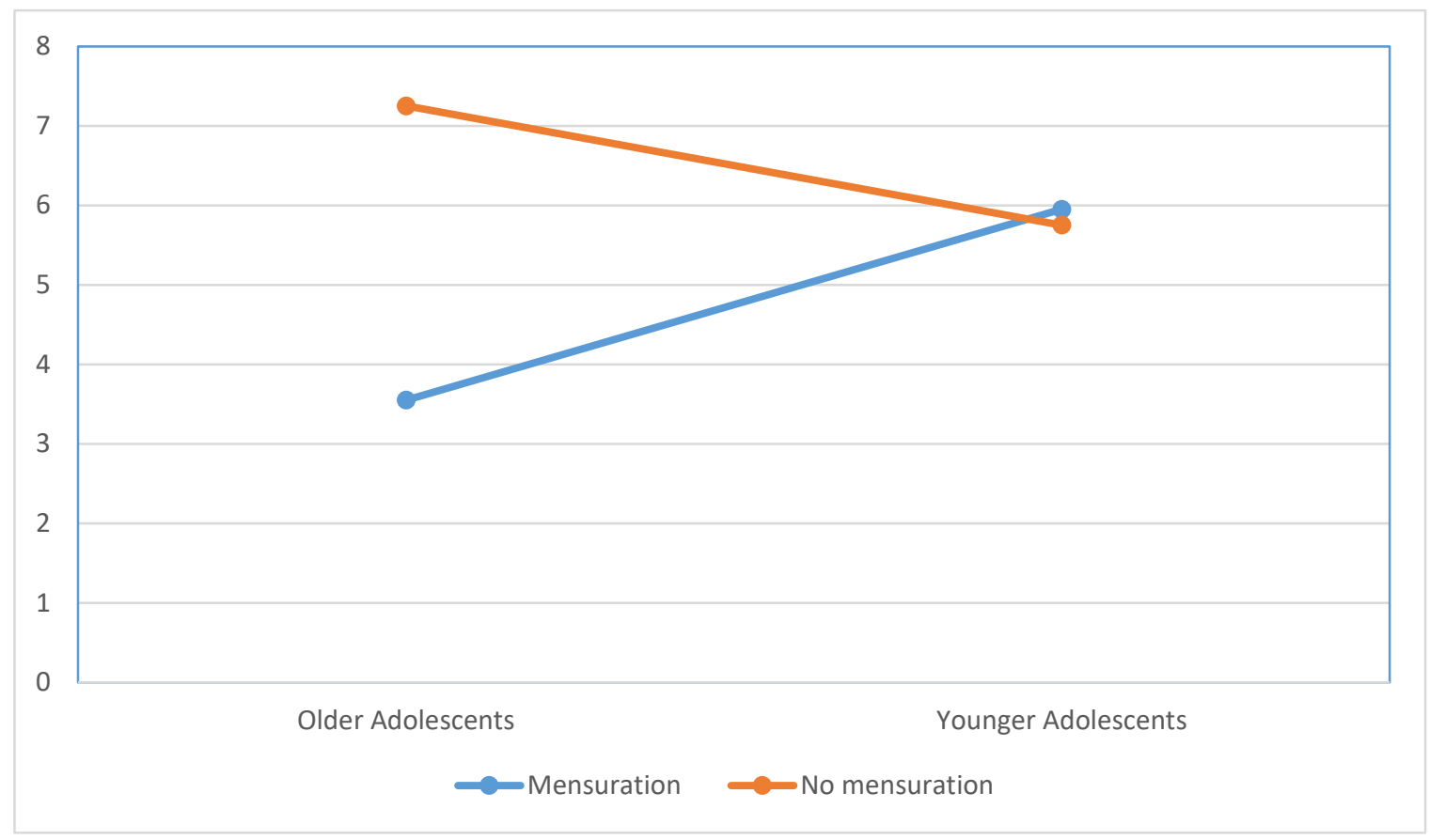

Figure 1: Interaction of reproductive maturity and age on serial recall.

\section{DISCUSSION}

The result of the study indicates significant influence of reproductive maturity on serial recall. This shows that there was a significant difference in serial recall between adolescents who had attained reproductive maturity and those who had not. Observation of the means indicate higher recall for none-mature participants; indicating less distraction and higher attention to the details of the environment.

The result of the study indicates non-significant influence of age on serial recall. This means that there was no significant difference in serial recall between younger adolescents and older adolescents. This reason for this result is attributable to the non-significant age gap between the two groups of adolescents in the study. Moreover, it possible that age differences in intelligence is waning with the high computer and mobile phone use among this adolescents today. Thus, development of the senses may not follow based on chronological age.

The result also indicates significant interaction of reproductive maturity and age on serial recall. Thus, observation of the mean matrix in Table 3 shows that the highest point of serial recall was went for older adolescents who were yet to attain reproductive maturity (see also figure 1). This result could be attributed to the lower level of distractions owing to the absence of pubertal stress experienced by adolescents who are yet to experience menstrual flow. It is expected that menstrual cycle with its attendant worries and surprises could divide the attention of a growing child, making her more aware of her body than her studies and social environment. 


\section{CONCLUSION}

This study has implications for eye-witness testimony and education. First, adolescents at puberty should be trained pay attention to their environment as well as their body. This is important because taking note of environmental changes may do well to protect the interest her has in keeping her body away from danger.

It also important to note that a less focused attention on reproductive development can help to improve concentration in academics. This is necessary given the rate of academic failure among many female senior secondary school students and female university undergraduates who initially were high flyers in primary and junior secondary school days.

\section{REFERENCES}

[1] Rehnman, J., \& Herlitz, A. (2007). Women remember more faces than men do. Acta Psychologica, 124(3), 344-355.

[2] Lindholm, T., \& Christianson, S. A. (1998). Gender effects in eyewitness accounts of a violent crime. Psychology. Crime \& Law, 4(4), 323-339.

[3] Rhodes, S., Greene, N. R., \& Naveh-Benjamin, M. (2019). Age Related Differences in Recall and Recognition: A MetaAnalysis. Retrieved on 16th April, 2019 from https://osf.io/5gx86/.

[4] Zelinski, E. M., Gilewski, M. J., \& Schaie, K. W. (1993). Individual differences in crosssectional and three-year longitudinal memory performance across the adult life span. Psychology and Aging, 8(2), 176-186.

[5] Herlitz, A., Nilsson, L.-G., \& Bäckman, L. (1997). Gender differences in episodic memory. Memory and Cognition, $25(6), 801-811$.

[6] Herlitz, A., \& Rehnman, J. (2008). Sex differences in episodic memory. Current Directions in Psychological Science, $17(1), 52-56$.

[7] Tulving, E. (1993). Human memory. In P. Andersen, O. Hvalby, O. Paulsen, \& B. Hökfelt (Eds.), Memory concepts 1993: Basic and clinical aspects (pp. 27-45). Amsterdam: Elsevier.

\section{APPENDIX}

List of Words

1. Peter

2. Car

3. Weeding

4. Table

5. 8 p.m.

6. Water

7. Johnpaul

8. Book

9. 10 p.m.

10. Okeke

11. Pencil

12. Emeka

13. Transcript

14. Examination

15. Cupboard

16. Gallery

17. Farming

18. Wallet

19. Shirt

20. Eunice 